\title{
Le Cercle et la spirale
}

Par Bronislaw Baczko

Après la lecture d'un nouveau travail de Jean Starobinski, ou après la relecture d'un de ses ouvrages plus anciens, je me plais assez souvent à reprendre les pages magistrales où il s'interroge lui-même sur sa méthode et médite sur le sens qu'il donne à la critique ${ }^{1}$. Je me laisse alors entraîner par la découverte de tout un jeu d'oppositions et de correspondances entre les deux discours, celui où le critique est au travail et celui qui porte sur le travail du critique. Quand Jean Starobinski nous offre son interprétation d'un texte, elle est tellement captivante et convaincante qu'elle semble s'imposer comme une sorte d'évidence. Parfois on se demande pourquoi il fallait attendre cette lecture pour que ces évidences se manifestent. La transparence de sa démarche critique est à la fois vraie et trompeuse; elle révèle et cache à la fois tout un travail, une élaboration combien complexe, qui soustend l'interprétation. Pour découvrir et comprendre ce travail, la démarche qu'il emprunte dans ses ouvrages, rien de plus lumineux que les pages méthodologiques déjà évoquées. Relues, elles s'éclairent du coup d'une lumière nouvelle, comme si leurs sens enfouis ne pouvaient être dégagés que par l'auteur lui-même, au cours de sa propre pratique interprétative. Autrement dit, une telle lecture parallèle nous offre une sorte de scène d'interprétation où les textes de Jean Starobinski se font face à face, se renvoient leurs lumières dans un jeu des miroirs, et ce spectacle est fascinant.

De ces pages d'une densité remarquable ne retenons qu'une seule question: comment le discours critique réunit-il, dans une seule et même démarche, l'approche structurale et la mise en perspective historique, comment l'une et l'autre, en fonction de leur pertinence, sont mises au service de la lecture critique du texte? A cette question dont il serait inutile de souligner l'importance pour les sciences humaines, Jean Starobinski apporte une réponse qui frappe par son originalité. Cela dit, nous n'avons guère l'intention de situer son œuvre par rapport aux interminables débats entre «historicisme» et «structuralisme», trop souvent aussi passionnés que stériles. A cette guerre entre histoire et structure que l'on nous annonçait maintes fois comme inévitable, il n'y croyait jamais et des «ismes» de toute sorte il s'en méfiait toujours. Avant toute réflexion méthodologique, son 
inquiétude intellectuelle et sa curiosité insatiable du phénomène humain le préservaient de leur langueur. Du fait que les sciences humaines ne se pratiquent plus aujourd'hui sans risques méthodologiques, Jean Starobinski en est parfaitement conscient. Ces risques, il les assume de son plein gré pour en faire autant de chances offertes au savoir et pour les transformer en exigence de rigueur. «Il faut rendre à son historicité non seulement la question méthodologique, non seulement l'objet de notre intérêt, mais notre intérêt même. Il ne me déplaît pas que le climat actuel y ramène notre attention, par l'obligation où il nous met de justifier nos choix, d'en réexaminer le bien-fondé, et de renouveler, en connaissance de cause, la confiance que nous portons non seulement à nos méthodes de travail, non seulement à nos objets d'étude, mais encore au sens même de l'étude». De son érudition prodigieuse et de son intelligence puissante, Jean Starobinski s'en sert pour planter des garde-fous contre la tentation de dogmatisme qui guette les «méthodologues» trop conquérants et, en fin de compte, assez ennuyeux. Lui, il ne s'enferme guère dans aucune «méthodologie» exclusive; il réfléchit sur le travail qu'il fait, le conceptualise et, du coup, nous appelle à réfléchir avec lui.

Ce n'est pas par hasard que sa réflexion sur «le scénario exemplaire de l'interprétation» vient après une analyse modèle d'une page de Rousseau (le célèbre épisode du «dîner de Turin», dans le livre III des Confessions). Tout se passe comme s'il nous faisait d'abord visiter, pièce après pièce, une superbe demeure; il nous laisse découvrir les paysages sur lesquels s'ouvrent les fenêtres, admirer l'intérieur, les collections qui égayent les pièces, ici un chefd'œuvre, ailleurs un simple bibelot. Au cours de cette visite on se sent de plus en plus chez soi dans cette maison solidement bâtie, merveilleusement aménagée, respirant la présence discrète et accueillante de son hôte. Ce n'est qu'ensuite qu'il nous montre la carte du site qu'il a choisi, le projet architectural qu'il a conçu, les matériaux qu'il a utilisés. A l'occasion il nous raconte également, avec la passion et le plaisir d'un vrai collectionnaire, où et comment il a trouvé tel ou tel objet rare, pourquoi l'avait-il exposé à un endroit précis qui le met en valeur et où il cadre le mieux avec l'ensemble. Après quoi, il ajoute, très sérieusement mais non sans un petit sourire, que, certes, on pourrait imaginer une autre architecture, bâtir une autre maison, la meubler différemment, tout en nous mettant en garde qu'il faut, surtout, respecter le site, construire sans bâclage et ne pas encombrer l'intérieur par la pacotille. On le croit, certes, puisqu'il le dit; on se demande pourtant si on y retrouvera ce sentiment, à la fois chaleureux et indéfinissable, d'être chez soi. 
Dans cette maison, on peut y entrer par plusieurs portes. Choississons celle de l'histoire, et suivons l'hôte qui s'est chargé de nous guider. «Ce que tout commence, dans les disciplines d'interprétation, par le choix d'un objet, et que ce choix n'est jamais l'effet du hasard: il s'est désigné à notre attention comme «devant-être-interprétés, comme «méritant-une-étude〉... L'objet à interpréter se désigne comme porteur de sens pour nous: il se désigne sur fond d'histoire, à nous, individus historiques. C'est l'histoire, derrière moi, en moi, l'histoire sous le nom de culture ou sous son aspect d'urgence actuelle, qui me donne une raison à m'intéresser à Rousseau, à sa révolte, à son écriture». Double historicité donc: celle de l'objet à étudier et celle du sujet qui fait le choix, et, du coup, affirme sa liberté dans l'histoire. La liberté et non pas l'arbitraire: un choix, certes, mais conscient des conditions présentes auxquelles il est soumis, un choix voué à faire croître l'entreprise du savoir, animé par la volonté de ne pas faire cèder à l'oubli les personnes, les événements, les œuvres issus du passé. Les deux projets, celui de l'historien et celui de l'interprète, se rejoignent jusqu'à se confondre dans cette volonté de comprendre le passé et de faire durer la mémoire. Ne croyons pas trop à tous ces cloisonnements institutionnels qui semblent les séparer, ne confondons pas la maîtrise de la technicité indispensable à tout savoir avec les objectifs que celui-ci se fixe. Toute recherche historique interprète le passé; toute enquête explicative est à la recherche des significations enfouies dans l'histoire. Interpréter, c'est exploiter «les réserves de sens possibles» dont sont riches les phénomènes du passé. «Le choix de l'objet à interpréter - que ce soit le Mö̈se de Michel-Ange, le coup d'Etat du 2 décembre, ou les Confessions - me propose dès le départ un phénomène global et concret, dont le sens pointe et préexiste, sous une forme déjà forte, aux explications dont je pourrai ultérieurement l'éclairer. Je pars donc d'une figure offerte, d'une forme discernée, d'un texte dont la signification à première vue est déjà assez puissante pour retenir mon attention et devenir le prétexte d'une enquête explicative, - d'une enquête ou d'une construction qui aura pour but de transformer la présignification en signification développée.»

«L'interprète et ses interpretenda se font donc face à face dans le temps historique.» Dans ce vis-à-vis précisément s'élabore tout un réseau de liaisons qui raccorde le critique à son objet et qui est traversé par le discours interprétatif. Les enjeux de cette élaboration sont multiples: le critique doit s'ouvrir au texte mais également le pénétrer; il doit l'incorporer dans son discours tout en l'affirmant dans son altérité; il doit le rapprocher tout en le tenant à distance; à la limite, il doit faire éclater le texte pour dégager les 
rapports entre ses données qui lui assurent sa structure propre (utilisons ce terme dans son sens le plus général et seulement pour indiquer l'orientation spécifique de l'entreprise critique: gagner en intelligibilité du texte en décelant les relations et les oppositions qui lui sont propres et à travers lesquelles s'affirme son unité qui n’en fait pas pour autant un «système»). Travail qui tient du paradoxe et on serait tenté de faire un parallèle entre le critique et le comédien, cet interprète par excellence, d'esquisser, dans le sillage de Diderot, un «paradoxe sur le critique». Travail qui aurait comme emblème un cercle, ou plutôt un double cercle à la fois tracé et parcouru par le discours interprétatif.

Assimilation du texte, donc «traduction, transcodage, ou plutôt transcription libre de quelques données mises en évidence à l'intérieur du texte». Ce transcodage, le critique l'effectue en se servant des instruments, du vocabulaire et du savoir, que lui fournissent les sciences humaines contemporaines. Pas de système formalisé de cette transcription; les sciences de l'homme tout en imposant au critique l'exigence de rigueur intellectuelle lui laisse pourtant un considérable espace de liberté. L'interprétation du texte relève ainsi à la fois de la science et de l'art, elle consiste, pour ainsi dire, dans l'emploi littéraire du savoir. Ce savoir demeure toujours trop général et trop abstrait par rapport à l'unicité du texte à interpréter. Le critique se laissera donc guider par un certain «flair», un peu à l'instar d'un clinicien chevronné. Lui aussi, tout en utilisant les techniques d'examen les plus modernes et les plus sophistiquées ne le multiplient pas pourtant à l'infini mais, à un moment, conclut au diagnostic grâce à son «talent clinique», une sorte de perception globale. Ce rapprochement de l'art de l'interprétation à la méthode clinique mériterait, certes, des développements et des nuances; l'analogie n'est valable que dans certaines limites. Si même ce rapprochement nous renvoie à la biographie de notre critique ne cédons pas pourtant à une pratique trop routinière: en guise de l'explication d'une œuvre conter la vie de son créateur. Le choix et le travail intellectuels du critique s'inscrivent, certes, dans un moment de sa vie mais ne s'expliquent pas entièrement par sa biographie. La recherche des antécédents voire des origines n'est plus la voie royale ni de l'histoire ni de l'interprétation. Celle-ci peut parfaitement explorer un champ historique sans épouser pourtant la forme narrative d'un récit auquel la succession chronologique des événements fournirait la trame, et sans caresser l'illusion de mettre ainsi au jour le lien causal par lequel ceuxci seraient réunis. Il se peut que l'interprétation gagne en rigueur et en pertinence en suspendant «toute hypothèse causale». N'insistons donc que 
sur «la description des évidences immanentes» à une telle entreprise critique et notamment sur la circularité du discours interprétatif.

Il est légitime que ce discours soit d'abord «indicatif de soi, qu'il s'affirme selon son style, son ordre et sa possibilité et que l'objet étudié lui soit l'occasion de prouver ses propres pouvoirs, ses qualités spécifiques - et qu'ainsi vienne au jour le langage de notre savoir (ou de notre conscience) dans sa particularité historique et dans sa visée de l'universel». En déployant son énergie assimilatrice, la parole interprétative réalise son propre projet: à la limite elle est tentée d'abolir toute «séparation entre sujet interprétant et objet interprété». Héritage lointain, peut-être, de l'exégèse religieuse au service de laquelle est apparue l'interprétation. L'exégète se trouve pourtant face à un seul texte, habité par la Parole, universelle et absolue, qui n'a qu'un seul sens englobant le monde et l'histoire. L'exégèse reste au fond un appel à l'assomption vers cette Parole, et quand elle est animée par un élan mystique, elle glisse vers la quête de l'illumination, de ce moment de transparence absolue du sujet communiquant directement avec le Verbe, sans passer par l'intermédiaire du texte. Le critique n'est pas un exégète. Il lui est d'avance acquis d'enquêter sur des textes dont chacun recèle plusieurs sens. Une seule lecture n'embrasse jamais cette pluralité, à la fois assurée et limitée par l'historicité du texte et du critique lui-même. Elaboré par le critique, son discours se prête en permanence à l'examen des ses propres conditions de possibilité, de ses fondements et de sa cohérence. Il ne gagne en transparence qu'en reconnaissant son obstacle, l'altérité du texte, et en acceptant son défi. Franchir cet obstacle, c'est fournir du travail approprié, faire profit de ses ressources et s'adapter à ses contours. L'obstacle ainsi aménagé se transforme en tronçon du chemin tracé et silloné par le projet critique. Ainsi le discours explicatif, en assimilant son objet, parcourt un cercle. La parole du critique est «origine et fin, mais n'accède à sa fin qu'après avoir traversé son objet... L'objet expliqué est pris en charge, il n'est pas seulement une illustration et un cas d'application d'une méthode préexistante, il devient partie intégrante du discours du savoir, il donne aux principes méthodologiques la possibilité de se transformer à travers une pratique, si bien qu'à la fin l'objet interprété constitue un élément nouveau du discours interprétant: il a cessé d'être une énigme à déchiffrer et devient à son tour un instrument de déchiffrement». A ce cercle de la parole qui se referme sur son origine, qui fait régner l'ordre du même, répond pourtant un autre parcours, lui aussi cirulaire mais prenant son origine tout à l'opposé: «un cercle qui va de l'objet à l'objet, qui part d'une occurence particulière, différente, 
significative, mais cette fois plus fortement légitimée dans sa particularité et sa signification». Envisagé dans cette perspective, le discours critique n'assimile plus son objet, mais c'est, au contraire, le texte qui appelle et absorbe l'interprétation. Ces deux «cercles» sont contemporains l'un de l'autre: élaborer l'interprétation, c'est les parcourir d'un mouvement doublement circulaire. Autrement dit, le travail critique aurait ainsi enrichi le texte par la parole explicative mais celle-ci aurait tiré tout son profit de l'exploration de la richesse du texte.

A la fin de cette aventure critique (mais est-elle jamais achevée?) nous retrouvons, certes, le même texte par lequel nous l'avons commencée. Nous le lirons pourtant autrement. Le critique nous l'a fait «visité» tel qu'il l'avait aménagé et enrichi: nous découvrons une structure cachée, des contextes inconnus, des sens enfouis, des dimensions historiques inaperçues. A la fin de cette visite le critique nous fait une confidence: au sortir de son expérience «le monde et la vie de l'interprète trouvent eux-mêmes un accroissement de sens». Nous le croyons d'autant plus volontiers qu'à travers son texte nous avons dialogué avec lui et, du coup, nous avons nous-mêmes bénéficié de cet accroissement.

La liste des lieux que Jean Starobinski nous a fait visiter serait longue et le $\mathrm{XVIII}^{\mathrm{e}}$ siècle y prend une place particulièrement importante: de Montesquieu à Rousseau, de Voltaire à Gibbon, de David à Goya, de la Flûte enchantée à la fête révolutionnaire. Ce serait une toute autre histoire que de s'interroger sur ce qui l'a amené à préférer ce site historique. Entre l'interprète et les œuvres interprétées il devrait y avoir, avant toute refléxion méthodologique, une première entente. Le «cercle» s'applique bien comme emblème au travail interprétatif sur un seul texte. Mais pour la longue série de textes critiques de Jean Starobinski il faudrait, peut-être, choisir un autre emblème; l'hélice qui s'enroule autour de l'axe que forme le siècle des Lumières. «L'interprétation, nous dit Jean Starobinski, veut tout ensemble l'abolition de la différence (par le discours inclusif et totalisateur) et le maintien à l'écart (par la compréhension de l'autre en tant que l'autre)». En ascendant avec lui la spirale interprétative nous ne cessions de découvrir le $\mathrm{XVIII}^{\mathrm{e}}$ siècle sous ses aspects nouveaux, souvent surprenants. Tout en le maintenant à l'écart, il nous l'a rapproché; tout en mettant en évidence sa spécificité historique, il en a fait une époque qui nous appartient un peu plus. Cette aventure qu'est la "visite guidée» du siècle des Lumières, ne nous enferme point dans le passé: nous en sortons par la même porte par laquelle nous y sommes entrés: celle de l'histoire et, partant, de notre propre 
historicité. Sans verser dans l'illusion, aussi facile que trompeuse, d'avoir «revécu» le siècle des Lumières nous l'avons mieux compris; lui gagnant en intelligibilité, nous en sommes un peu plus éclairés. Grâce à notre guide cette visite n'était pas un simple divertissement mais surtout un apprentissage: d'un texte à l'autre, nous ne cessions d'apprendre l'art de lecture. Apprentis lecteurs, nous nous promettons de revenir, avec notre guide, sur les lieux déjà visités ensemble mais où il nous reste un tas de choses à découvrir. Nous nous réjouissons surtout de pouvoir continuer cet apprentissage, de suivre le guide sur les nouveaux parcours qu'il choisira pour lui-même et, du coup, nous en fera don.

\section{Note}

1 Jean Starobinski, La relation critique, Paris, Gallimard, pp.158-169. Dans la suite, toutes nos citations sont empruntées de ces pages.

Prof. Bronislaw Baczko 10, avenue du Lignon CH-1219 Genève 